\title{
Assessment of Lesbian, Gay, Bisexual, Transgender, and Questioning Experiences Within a Large Southeast Training Program
}

\section{Zachary W. Walker ( $\sim$ Zwalker1@bwh.harvard.edu )}

Brigham and Women's Hospital

\section{Mary Appah}

University of Alabama at Birmingham

Inmaculada Aban

University of Alabama at Birmingham

Brenessa M. Lindeman

University of Alabama at Birmingham

Latesha E. Elopre

University of Alabama at Birmingham

Alice R. Goepfert

University of Alabama at Birmingham

Samantha V. Hill

University of Alabama at Birmingham

\section{Research Article}

Keywords: LGBTQ, Physician, Awareness, Environment

Posted Date: September 7th, 2021

DOl: https://doi.org/10.21203/rs.3.rs-803339/v1

License: (9) (1) This work is licensed under a Creative Commons Attribution 4.0 International License. Read Full License 


\section{Abstract \\ Background}

The United States has become increasingly diverse resulting in greater strides to improve workforce diversity and inclusivity.

\section{Objective}

The objective of this study is to identify trainees in Graduate Medical Education who identify as Lesbian, Gay, Bisexual, Transgender or Questioning (LGBTQ) and compare their experiences as a trainee to non-LGBTQ trainees within the medical workplace.

\section{Methods}

We conducted a cross-sectional, exploratory survey from December 1, 2020 to January 14, 2021 at a single, large teaching institution. We collected data anonymously and stored it in a REDCap database. We excluded surveys in which trainees did not respond to sexual orientation. We used contingency tables and Fisher's exact test to identify outcomes associated with sexual orientation and gender identity particularly with regard to professionalism, wellbeing, and satisfaction with training.

\section{Results}

We distributed the survey to 840 trainees. 730 trainees were included with 23 (3.2\%) self-identifying as LGBTQ and 707 (96\%) as Straight. LGBTQ trainees were more likely to experience offensive remarks based on race/ethnicity ( $p$ $=0.03$ ) and sexual orientation $(p=0.01)$. Secondary analysis based on race found that Blacks and Other were more likely to report differences based on professionalism and satisfaction with their training program. There was no difference seen among LGBTQ trainees based on race.

\section{Conclusion}

We found trainees who identified as LGBTQ were more likely to experience discrimination/microaggressions. Also, underrepresented minorities in medicine were more likely to encounter discrimination and dissatisfaction with their training. More efforts are needed in academics to promote safe and supportive LGBTQ and minority training experiences.

\section{Introduction}

The United States has become increasingly diverse in relation to race, ethnicity, and sexual and gender identity, resulting in greater strides to improve workforce diversity and inclusivity. In 2009, the Liaison Committee on Medical Education and the Association of American Medical Colleges (AAMC) created recommendations promoting the accessibility of medical education to diverse applicants and improving representation of underrepresented individuals in medicine (URiM) relative to their numbers in the general population. ${ }^{1,2}$ 
Approximately $3.5 \%$ of Americans identify as lesbian, gay, or bisexual; and $0.3 \%$ identify as transgender. ${ }^{3}$ However, it is currently unclear how many healthcare physicians and trainees identify as lesbian, gay, bisexual, transgender, and questioning (LGBTQ) making quantification of optimal diversity among Graduate Medical Education (GME) trainees regarding sexual orientation and gender identity to better reflect national demographics as well as patient population needs difficult.

Keeping with the AAMC guidelines, LGBTQ trainees must be afforded the opportunity to learn and practice in welcoming environments that are responsive to their professional needs. However, members of the LGBTQ community working in healthcare are faced with numerous challenges, (e.g. lack of mentorship, fear of discrimination/harassment, lack of promotion within the workforce). ${ }^{4,5}$ Due to these circumstances, it is also difficult for academic institutions to retain LGBTQ residents, fellows, medical students, and faculty, especially those who may not be "open" about their sexual orientation or gender identity. LGBTQ physicians and trainees may experience diminished interest in academic careers and report lower career satisfaction as faculty due to discriminatory and negative experiences during their training. ${ }^{4,5}$ In addition, LGBTQ individuals who conceal their identity have increased rates of depression and anxiety which ultimately translates to an inability to focus on their academics or their clinical performance. ${ }^{6,7}$ Thus, the overall well-being of LGBTQ trainees and physicians is perceived as poor.

In Alabama, 3.1\% of adults identify as LGBTQ however, the percent of LGBTQ trainees and physicians, as well as their well-being, is unknown at the largest academic medical center in Alabama because no standardized, routine way of collecting sexual orientation and gender identity data for GME trainees exists. ${ }^{8}$ Insights gained from such an evaluation can also be applied to other university-affiliated hospitals complying with AAMC guidelines to improve diversity and working environments for LGBTQ trainees. This study site is an active participant in the Healthcare Equality Index (HEI) survey- a "benchmark tool that evaluates healthcare facilities' policies and practices related to the equity and inclusion of their LGBTQ patients, visitors and employees". ${ }^{9}$ The study site is recognized as a 2020 LGBTQ Healthcare Equality Leader; however, there is no benchmark to represent training equality for LGBTQ trainees.

The Positive emotion, Engagement, Relationships, Meaning, and Accomplishment (PERMA) theory of well-being provides this study's theoretical basis ${ }^{10}$. PERMA suggests alleviation of suffering does not equate to flourishing. ${ }^{10}$ For example, distributing LGBTQ paraphernalia may alleviate the thought of feeling unwelcome, but does not allow LGBTQ trainees to thrive in a department that has unconscious bias towards LGBTQ individuals. Also embedded in this study is intersectionality (Fig. 1).

Intersectionality is a framework to explore how multiple parts of one's identity (e.g. sexuality, race, gender) interact to impact systems. Thus, the focus of well-being should be on how best to allow individuals to flourish in their environment and not solely on diminishing harm. Gaining a better understanding of the current lived experiences of LGBTQ trainees working in academic institutions and how intersectionalities influence their wellbeing is crucial in developing targeted interventions focused on helping trainees flourish academically and clinically in their environments as well as for the health of the patients they serve.

Thus, the objective of this exploratory study is to identify GME trainees who identify as LGBTQ and ascertain their experiences as a trainee and healthcare provider at a single, large teaching institution

\section{Methods}




\section{Study design and participants}

We conducted a cross-sectional, exploratory study of data collected from GME trainees (residents and fellows) at the largest academic training center in AL using An 80-item survey, deployed annually since 2015 assessing the work and learning environment among trainees. include six sections (background information, professionalism, well-being, work experience, satisfaction with training program, and LGBTQ awareness). In conjunction with the PERMA theory, we equated professionalism to relationships, well-being to positive emotion, work experience to meaning, satisfaction with training program to engagement, and LGBTQ awareness to accomplishment (Fig. 1). We measured and explored each of these components independently. The scores in any of these elements provides insight regarding the degree of well-being, or flourishing, trainees may have at the institution. In addition, we believe intersectionality, especially among LGBTQ individuals, provides another barrier among those faced with unsupportive environments and should be included in the evaluation using this theory.

This self-administered survey was distributed to all GME trainees between December 1, 2020 to January 14, 2021. Inclusion criteria: a university GME resident or fellow during the time the survey was dispersed. Exclusion criterion: any trainee who did not identify their sexual orientation in the survey. Participants were recruited via email using the GME listserv. The estimated completion time was approximately 10 minutes and was uncompensated. All data was collected anonymously and stored in a REDCap database. This study was approved by the Institutional Review Board at the University of Alabama at Birmingham.

\section{Outcomes of Interest}

Seven questions about LGBTQ awareness were added (Addendum A). These questions were generated based on internal institutional review committee feedback focused on LGBTQ quality and prior studies with similar assessments. ${ }^{11,12}$ LGBTQ individuals were defined based on sexual orientation, with individuals identifying as transsexual being included in LGBTQ.

\section{Statistical Analysis}

Descriptive statistics were used to summarize patient characteristics. Contingency tables and Fisher's exact test were performed to identify outcomes associated with sexual orientation and gender identity particularly regarding professionalism, well-being, and satisfaction with training. Data reported in this study reflects statistical significance when $p<0.05$. Given the small cell size in primary analysis of our secondary and tertiary objectives, we ran a separate analysis with collapsed variables. All outcomes were collapsed to the following: agree (slightly agree, moderately agree, and strongly agree); disagree (slightly disagree, moderately disagree, strongly disagree, or neutral); No (never); Yes (once, occasionally, and frequently); important (very important, moderately important, and somewhat important); and not important (less important, not important at all, and I'm not sure). All analyses were performed using R software, version 4.0.2 (R Core Team, 2021).

\section{Results}

Eight hundred and forty GME trainees took the survey. 730 trainees met inclusion criteria: 707 (96\%) Straight trainees and 23 (3.2\%) LGBTQ trainees (Fig. 1). There were no significant differences between the two groups based on age, postgraduate year (PGY) level, American College of Graduate Medical Education (ACGME) category, and relationship status. There were differences in gender $(p=<0.01)$ and race $(p=0.01)$ between the two groups with the Straight cohort containing more White/Caucasian, cis-gendered males (52\% vs 72\%) (Table 1). We did not 
include these characteristics as confounders in our analysis given the small sample size, the inherent bias based in the cohorts, and the overall similarity within the general medical population. 
Table 1

Baseline demographics

\begin{tabular}{|c|c|c|c|c|c|}
\hline Characteristics & & $\mathbf{N}$ & LGBTQ N(\%) & Straight N(\%) & $P$ value \\
\hline \multirow[t]{4}{*}{ Age } & $18-24$ & 3 & $1(4.3)$ & $2(0.3)$ & \\
\hline & $25-34$ & 722 & $20(87)$ & $631(89.3)$ & \\
\hline & $35+$ & 87 & $2(8.7)$ & $72(10.2)$ & \\
\hline & $N A^{*}$ & 28 & $0(0)$ & $2(0.3)$ & 0.11 \\
\hline \multirow[t]{8}{*}{ PGY level } & PGY1 & 135 & $5(21.7)$ & $115(16.3)$ & \\
\hline & PGY2 & 146 & $5(21.7)$ & 132(18.7) & \\
\hline & PGY3 & 172 & $3(13)$ & $148(20.9)$ & \\
\hline & PGY4 & 157 & $8(34.8)$ & $126(17.8)$ & \\
\hline & PGY5 & 105 & $1(4.3)$ & $98(13.9)$ & \\
\hline & PGY6 & 67 & $0(0)$ & $61(8.6)$ & \\
\hline & PGY7 or higher & 28 & $1(4.3)$ & $24(3.4)$ & \\
\hline & $N A^{*}$ & 30 & $0(0)$ & $3(0.4)$ & 0.21 \\
\hline \multirow[t]{5}{*}{ ACGME Category } & Dentistry & 17 & $0(0)$ & $11(1.6)$ & \\
\hline & Hospital-based & 195 & $4(17.4)$ & $176(24.9)$ & \\
\hline & Medical & 427 & $11(47.8)$ & $353(49.9)$ & \\
\hline & Surgical & 201 & $8(34.8)$ & 167(23.6) & \\
\hline & $N A^{*}$ & 0 & $0(0)$ & $0(0)$ & 0.64 \\
\hline \multirow[t]{7}{*}{ Gender } & Male & 462 & $11(47.8)$ & $402(56.9)$ & \\
\hline & Female & 342 & $10(43.5)$ & $299(42.3)$ & \\
\hline & Transgender (male to female) & 1 & $1(4.3)$ & $0(0)$ & \\
\hline & Transgender (female to male) & 1 & $1(4.3)$ & $0(0)$ & \\
\hline & Non-binary & 1 & $0(0)$ & $0(0)$ & \\
\hline & Not listed & 4 & $0(0)$ & $3(0.4)$ & \\
\hline & $N A^{*}$ & 29 & $0(0)$ & $3(0.4)$ & $<0.01$ \\
\hline \multirow[t]{3}{*}{ Race } & White/Caucasian & 523 & $12(52.5)$ & $509(72)$ & \\
\hline & Black/African-American/African & 32 & $4(17.4)$ & $27(3.8)$ & \\
\hline & Asian & 109 & $3(13)$ & 103(14.6) & \\
\hline
\end{tabular}

LGBTQ = Lesbian, Gay, Bisexual, Transgender, and Questioning; NA = Not answered; PGY = postgraduate year; ACGME = Accreditation Council of General Medical Education

*Not included in the calculation of the p-value from the Fisher's exact test. 


\begin{tabular}{|llllll}
\hline Characteristics & & N & LGBTQ N(\%) & Straight N(\%) & P value \\
\hline & Other & 71 & $4(17.4)$ & $64(9.1)$ & \\
& NA* & 105 & $0(0)$ & $4(0.6)$ & 0.01 \\
\hline Relationship status & Never married & 297 & $12(52.2)$ & $255(36.1)$ & \\
& Married or Domestic partnership & 492 & $11(47.8)$ & $434(61.4)$ & \\
& Widowed & 1 & $0(0)$ & $1(0.1)$ & \\
& Divorced & 13 & $0(0)$ & $13(1.8)$ & \\
& Separated & 4 & $0(0)$ & $3(0.4)$ & \\
\hline & NA* & 33 & $0(0)$ & $1(0.1)$ & \\
\hline
\end{tabular}

Analysis of the LGBTQ-specific questions of the survey revealed the majority of individuals identified as Straight (707 (84\%)), Gay (10 (1\%)), and Bisexual (8 (1\%)) and preferred either she/her (309 (37\%)) or he/him (409 (49\%)) (Table 2). Only $0.1 \%$ of trainees reported their department being aware of their gender identity. A majority of individuals agreed the institution (551 (66\%)) and department (589 (70\%)) have a welcoming LGBTQ environment. Lastly, only $0.1 \%$ of trainees responded to being willing to mentor someone who identifies as LGBTQ. All respondents willing to mentor LGBTQ individuals identified as LGBTQ (data not shown).

\section{Table 2: LGBTQ-specific questions}




\begin{tabular}{|c|c|c|}
\hline Questions & & $\mathrm{N}(\%)$ \\
\hline In terms of sexual orientation, what do you think of yourself as (please check all & Straight & $707(84.2)$ \\
\hline & Gay & $10(1.2)$ \\
\hline & Lesbian & $1(0.1)$ \\
\hline & Bisexual & $8(1)$ \\
\hline & Ques & $1(0.1)$ \\
\hline & Asexual & $0(0)$ \\
\hline & Pansexual & $3(0.4)$ \\
\hline & NA & $110(13.1)$ \\
\hline What are your gender pronouns? & She/her & $309(36.8)$ \\
\hline & $\mathrm{He} / \mathrm{him}$ & $409(48.7)$ \\
\hline & They/them & $0(0)$ \\
\hline & $\begin{array}{l}\text { Not listed } \\
\text { above }\end{array}$ & $7(0.8)$ \\
\hline & NA & 115(13.7) \\
\hline Is your department aware of your gender identity? & Yes & $1(0.1)$ \\
\hline & No & $1(0.1)$ \\
\hline & NA & 838(99.8) \\
\hline The institution has a welcoming LGBTQ + environment. & Strongly Agree & 233(27.7) \\
\hline & Agree & $318(37.9)$ \\
\hline & Neutral & 175(20.8) \\
\hline & Disagree & $6(0.7)$ \\
\hline & $\begin{array}{l}\text { Strongly } \\
\text { Disagree }\end{array}$ & $0(0)$ \\
\hline & NA & 108(12.9) \\
\hline My department has a welcoming LGBTQ + environment. & Strongly Agree & $287(34.2)$ \\
\hline & Agree & $302(36)$ \\
\hline & Neutral & 138(16.4) \\
\hline & Disagree & $4(0.5)$ \\
\hline & $\begin{array}{l}\text { Strongly } \\
\text { Disagree }\end{array}$ & $0(0)$ \\
\hline & NA & 109(13.0) \\
\hline Would you be willing to mentor someone who identifies as LGBTQ+? & Yes & $1(0.1)$ \\
\hline
\end{tabular}


No

NA

LGBTQ = Lesbian, Gay, Bisexual, Transgender, and Questioning; NA = Not answered

Table 3 reveals findings pertaining to professionalism, well-being, and satisfaction with training programs.

Significant differences in experiences with professionalism were found; LGBTQ trainees were more likely to report being subjected to racially or ethnically offensive remarks/names $(p=0.03)$ and being subjected to offensive remarks/names related to sexual orientation $(p=0.01)$. There was no evidence of difference found based on wellbeing or satisfaction with the training program between LGBTQ and Straight trainees. 
Table 3

Analysis of responses to questions regarding professionalism, well-being, and satisfaction with training program between Lesbian, Gay, Bisexual, Transgender, and Questioning (LGBTQ) and Straight trainees.

\begin{tabular}{|c|c|c|c|c|c|}
\hline & & $\mathbf{N}$ & $\begin{array}{l}\text { LGBTQ } \\
\text { N(\%) }\end{array}$ & $\begin{array}{l}\text { Straight } \\
\mathrm{N}(\%)\end{array}$ & $\begin{array}{l}P \\
\text { value }\end{array}$ \\
\hline \multicolumn{6}{|l|}{ Professionalism } \\
\hline \multirow[t]{5}{*}{ Been publicly humiliated? } & Never & 691 & $23(100)$ & 624(88.3) & \\
\hline & Once & 46 & $0(0)$ & $44(6.2)$ & \\
\hline & Occasionally & 43 & $0(0)$ & $31(4.4)$ & \\
\hline & Frequently & 5 & $0(0)$ & $4(0.6)$ & \\
\hline & $N A^{*}$ & 55 & $0(0)$ & $4(0.6)$ & 0.53 \\
\hline \multirow[t]{5}{*}{ Been threatened with physical harm? } & Never & 763 & $23(100)$ & 684(96.7) & \\
\hline & Once & 12 & $0(0)$ & $12(1.7)$ & \\
\hline & Occasionally & 8 & $0(0)$ & $6(0.8)$ & \\
\hline & Frequently* & 0 & $0(0)$ & $0(0)$ & \\
\hline & $N A^{*}$ & 57 & $0(0)$ & $5(0.7)$ & $\begin{array}{l}> \\
0.99\end{array}$ \\
\hline \multirow[t]{5}{*}{ Been physically harmed? } & Never & 778 & $23(100)$ & 697(98.6) & \\
\hline & Once & 6 & $0(0)$ & $5(0.7)$ & \\
\hline & Occasionally* & 0 & $0(0)$ & $0(0)$ & \\
\hline & Frequently* & 0 & $0(0)$ & $0(0)$ & \\
\hline & $N A^{*}$ & 56 & $0(0)$ & $5(0.7)$ & $\begin{array}{l}> \\
0.99\end{array}$ \\
\hline \multirow[t]{5}{*}{ Been subjected to unwanted sexual advances? } & Never & 758 & $22(95.7)$ & $680(96.2)$ & \\
\hline & Once & 15 & $0(0)$ & 13(1.8) & \\
\hline & Occasionally & 11 & $1(4.3)$ & $9(1.3)$ & \\
\hline & Frequently* & 0 & $0(0)$ & $0(0)$ & \\
\hline & $N A^{*}$ & 56 & $0(0)$ & $5(0.7)$ & 0.32 \\
\hline \multirow[t]{4}{*}{ Been subjected to offensive sexist remarks/names? } & Never & 712 & $21(91.3)$ & $643(90.9)$ & \\
\hline & Once & 26 & $0(0)$ & 23(3.3) & \\
\hline & Occasionally & 43 & $2(8.7)$ & $34(4.8)$ & \\
\hline & Frequently & 3 & $0(0)$ & $2(0.3)$ & \\
\hline
\end{tabular}

LGBTQ = Lesbian, Gay, Bisexual, Transgender, and Questioning; NA = Not answered

*Not included in the calculation of the p-value from the Fisher's exact test. 


\begin{tabular}{|c|c|c|c|c|c|}
\hline & & N & $\begin{array}{l}\text { LGBTQ } \\
\mathrm{N}(\%)\end{array}$ & $\begin{array}{l}\text { Straight } \\
\mathrm{N}(\%)\end{array}$ & $\begin{array}{l}P \\
\text { value }\end{array}$ \\
\hline & $N A^{*}$ & 56 & $0(0)$ & $5(0.7)$ & 0.47 \\
\hline \multirow{5}{*}{$\begin{array}{l}\text { Been denied opportunities for training or rewards } \\
\text { based on gender? }\end{array}$} & Never & 756 & $22(95.7)$ & 681(96.3) & \\
\hline & Once & 12 & $0(0)$ & 11(1.6) & \\
\hline & Occasionally & 13 & $1(4.3)$ & $8(1.1)$ & \\
\hline & Frequently & 3 & $0(0)$ & $2(0.3)$ & \\
\hline & $N A^{*}$ & 56 & $0(0)$ & $5(0.7)$ & 0.33 \\
\hline \multirow{5}{*}{$\begin{array}{l}\text { Been subjected racially or ethnically offensive } \\
\text { remarks/names? }\end{array}$} & Never & 718 & 19(82.6) & 648(91.7) & \\
\hline & Once & 30 & $0(0)$ & $27(3.8)$ & \\
\hline & Occasionally & 33 & $4(17.4)$ & $25(3.5)$ & \\
\hline & Frequently & 1 & $0(0)$ & $1(0.1)$ & \\
\hline & $N A^{*}$ & 58 & $0(0)$ & $6(0.8)$ & 0.03 \\
\hline \multirow{5}{*}{$\begin{array}{l}\text { Been denies opportunities for training or rewards } \\
\text { based on race or ethnicity? }\end{array}$} & Never & 766 & 22(95.7) & $687(97.2)$ & \\
\hline & Once & 7 & $0(0)$ & $6(0.8)$ & \\
\hline & Occasionally & 6 & $0(0)$ & $6(0.8)$ & \\
\hline & Frequently & 1 & $0(0)$ & $1(0.1)$ & \\
\hline & $N A^{*}$ & 60 & $1(4.3)$ & $7(1.0)$ & $>0.99$ \\
\hline \multirow{5}{*}{$\begin{array}{l}\text { Been subjected to offensive remarks/names related } \\
\text { to sexual orientation? }\end{array}$} & Never & 775 & $21(91.3)$ & 698(98.7) & \\
\hline & Once & 4 & $1(4.3)$ & $3(0.4)$ & \\
\hline & Occasionally & 3 & $1(4.3)$ & $1(0.1)$ & \\
\hline & Frequently & 1 & $0(0)$ & $0(0)$ & \\
\hline & $N A^{*}$ & 57 & $0(0)$ & $5(0.7)$ & 0.01 \\
\hline \multirow{4}{*}{$\begin{array}{l}\text { Been denied opportunities for training or rewards } \\
\text { based on sexual orientation? }\end{array}$} & Never & 780 & 22(95.7) & $700(99.0)$ & \\
\hline & Once & 2 & $1(4.3)$ & $1(0.1)$ & \\
\hline & Occasionally* & 0 & $0(0)$ & $0(0)$ & \\
\hline & Frequently* & 0 & $0(0)$ & $0(0)$ & \\
\hline
\end{tabular}

LGBTQ = Lesbian, Gay, Bisexual, Transgender, and Questioning; NA = Not answered

*Not included in the calculation of the p-value from the Fisher's exact test. 


\begin{tabular}{|c|c|c|c|c|c|}
\hline & & N & $\begin{array}{l}\text { LGBTQ } \\
\mathrm{N}(\%)\end{array}$ & $\begin{array}{l}\text { Straight } \\
\mathrm{N}(\%)\end{array}$ & $\begin{array}{l}P \\
\text { value }\end{array}$ \\
\hline & $N A^{*}$ & 58 & $0(0)$ & $6(0.8)$ & 0.06 \\
\hline \multirow[t]{5}{*}{ Shouting, use of belittling or abusive language } & Never & 632 & $21(91.3)$ & $577(81.6)$ & \\
\hline & Once & 63 & $1(4.3)$ & $59(8.3)$ & \\
\hline & Occasionally & 70 & $0(0)$ & $62(8.8)$ & \\
\hline & Frequently & 6 & $1(4.3)$ & $4(0.6)$ & \\
\hline & $N A^{*}$ & 69 & $0(0)$ & $5(0.7)$ & 0.10 \\
\hline \multirow{5}{*}{$\begin{array}{l}\text { Verbal, written, or physical personal attacks directed } \\
\text { at others }\end{array}$} & Never & 705 & $20(87.0)$ & $649(91.8)$ & \\
\hline & Once & 27 & $1(4.3)$ & 22(3.1) & \\
\hline & Occasionally & 36 & $2(8.7)$ & $29(4.1)$ & \\
\hline & Frequently & 4 & $0(0)$ & $3(0.4)$ & \\
\hline & $N A^{*}$ & 68 & $0(0)$ & $4(0.6)$ & 0.36 \\
\hline \multirow{5}{*}{$\begin{array}{l}\text { Public derogatory comments about quality of care } \\
\text { by other professionals }\end{array}$} & Never & 631 & $20(87)$ & $582(82.3)$ & \\
\hline & Once & 34 & $0(0)$ & $32(4.5)$ & \\
\hline & Occasionally & 104 & $3(13)$ & 88(12.4) & \\
\hline & Frequently & 4 & $0(0)$ & $3(0.4)$ & \\
\hline & $N A^{*}$ & 67 & $0(0)$ & $2(0.3)$ & 0.83 \\
\hline \multirow{5}{*}{$\begin{array}{l}\text { Inappropriate expressions of anger (e.g., throwing } \\
\text { things, destruction of property) }\end{array}$} & Never & 738 & $23(100)$ & $673(95.2)$ & \\
\hline & Once & 18 & $0(0)$ & $17(2.4)$ & \\
\hline & Occasionally & 11 & $0(0)$ & $9(1.3)$ & \\
\hline & Frequently & 2 & $0(0)$ & $2(0.3)$ & \\
\hline & $N A^{*}$ & 71 & $0(0)$ & $6(0.8)$ & $\begin{array}{l}> \\
0.99\end{array}$ \\
\hline \multirow[t]{5}{*}{ Physical assault } & Never & 770 & $23(100)$ & 702(99.3) & \\
\hline & Once & 1 & $0(0)$ & $1(0.1)$ & \\
\hline & Occasionally* & 0 & $0(0)$ & $0(0)$ & \\
\hline & Frequently* & 0 & $0(0)$ & $0(0)$ & \\
\hline & $N A^{*}$ & 69 & $0(0)$ & $4(0.6)$ & $\begin{array}{l}> \\
0.99\end{array}$ \\
\hline \multicolumn{6}{|c|}{ LGBTQ = Lesbian, Gay, Bisexual, Transgender, and Questioning; NA = Not answered } \\
\hline
\end{tabular}




\begin{tabular}{|c|c|c|c|c|c|}
\hline & & $\mathbf{N}$ & $\begin{array}{l}\text { LGBTQ } \\
\mathrm{N}(\%)\end{array}$ & $\begin{array}{l}\text { Straight } \\
\mathrm{N}(\%)\end{array}$ & $\begin{array}{l}\mathrm{P} \\
\text { value }\end{array}$ \\
\hline \multirow{5}{*}{$\begin{array}{l}\text { Unwelcome or wanted physical advances of a sexual } \\
\text { nature }\end{array}$} & Never & 762 & $23(100)$ & $694(98.2)$ & \\
\hline & Once & 4 & $0(0)$ & $4(0.6)$ & \\
\hline & Occasionally & 5 & $0(0)$ & $5(0.7)$ & \\
\hline & Frequently* & 0 & $0(0)$ & $0(0)$ & \\
\hline & $N A^{*}$ & 69 & $0(0)$ & $4(0.6)$ & $\overrightarrow{0.99}$ \\
\hline \multirow[t]{5}{*}{ Verbal abuse or joking based on gender } & Never & 712 & 21(91.3) & $655(92.6)$ & \\
\hline & Once & 20 & $0(0)$ & $18(2.5)$ & \\
\hline & Occasionally & 36 & $1(4.3)$ & $28(4.0)$ & \\
\hline & Frequently & 1 & $0(0)$ & $1(0.1)$ & \\
\hline & $N A^{*}$ & 71 & $1(4.3)$ & $5(0.7)$ & 0.78 \\
\hline \multirow[t]{5}{*}{ Verbal abuse or joking based on race/ethnicity } & Never & 735 & $20(87.0)$ & 673(95.2) & \\
\hline & Once & 13 & $2(8.7)$ & $11(1.6)$ & \\
\hline & Occasionally & 21 & $1(4.3)$ & $17(2.4)$ & \\
\hline & Frequently & 1 & $0(0)$ & $1(0.1)$ & \\
\hline & $N A^{*}$ & 70 & $0(0)$ & $5(0.7)$ & 0.08 \\
\hline \multirow[t]{5}{*}{ Verbal abuse or joking based on sexual orientation } & Never & 754 & $23(100)$ & $688(97.3)$ & \\
\hline & Once & 7 & $0(0)$ & $7(1.0)$ & \\
\hline & Occasionally & 9 & $0(0)$ & $7(1.0)$ & \\
\hline & Frequently* & 0 & $0(0)$ & $0(0)$ & \\
\hline & $N A^{*}$ & 70 & $0(0)$ & $5(0.7)$ & $>_{0.99}$ \\
\hline \multicolumn{6}{|l|}{ Well-Being } \\
\hline \multirow[t]{3}{*}{ Have you felt emotionally drained from your work? } & Yes & 424 & $10(43.5)$ & $310(55.9)$ & \\
\hline & No & 331 & $13(56.5)$ & $395(43.8)$ & \\
\hline & $N A^{*}$ & 85 & $0(0)$ & $2(0.3)$ & 0.29 \\
\hline \multirow[t]{2}{*}{$\begin{array}{l}\text { Have you worried that your work is hardening you } \\
\text { emotionally? }\end{array}$} & Yes & 329 & $8(34.8)$ & $307(43.4)$ & \\
\hline & No & 428 & $15(65.2)$ & $400(56.6)$ & \\
\hline
\end{tabular}

LGBTQ = Lesbian, Gay, Bisexual, Transgender, and Questioning; NA = Not answered

*Not included in the calculation of the p-value from the Fisher's exact test. 


\begin{tabular}{|c|c|c|c|c|c|}
\hline & & $N$ & $\begin{array}{l}\text { LGBTQ } \\
\mathrm{N}(\%)\end{array}$ & $\begin{array}{l}\text { Straight } \\
\mathrm{N}(\%)\end{array}$ & $\begin{array}{l}P \\
\text { value }\end{array}$ \\
\hline & $N A^{*}$ & 83 & $0(0)$ & $0(0)$ & 0.52 \\
\hline \multirow{3}{*}{$\begin{array}{l}\text { Have you often been bothered by feeling down, } \\
\text { depressed, or hopeless? }\end{array}$} & Yes & 189 & $6(26.1)$ & $175(24.8)$ & \\
\hline & No & 565 & $17(73.9)$ & $529(74.8)$ & \\
\hline & $N A^{*}$ & 86 & $0(0)$ & $3(0.4)$ & 0.81 \\
\hline \multirow{3}{*}{$\begin{array}{l}\text { Have you fallen asleep while sitting inactive in a } \\
\text { public place? }\end{array}$} & Yes & 138 & $2(8.7)$ & $132(18.7)$ & \\
\hline & No & 618 & $21(91.3)$ & $574(81.2)$ & \\
\hline & $N A^{*}$ & 84 & $0(0)$ & $1(0.1)$ & 0.28 \\
\hline \multirow{3}{*}{$\begin{array}{l}\text { Have you felt that all things you had to do were } \\
\text { piling up so high that you could not overcome them? }\end{array}$} & Yes & 210 & $10(43.5)$ & 192(27.2) & \\
\hline & No & 545 & $13(56.5)$ & $514(72.7)$ & \\
\hline & $N A^{*}$ & 85 & $0(0)$ & $1(0.1)$ & 0.10 \\
\hline \multirow{3}{*}{$\begin{array}{l}\text { Have you been bothered by emotional problems } \\
\text { (such as feeling anxious, depressed, or irritable)? }\end{array}$} & Yes & 312 & $10(43.5)$ & 294(41.6) & \\
\hline & No & 442 & $13(56.5)$ & $410(58.0)$ & \\
\hline & $N A^{*}$ & 86 & $0(0)$ & $3(0.4)$ & $\overrightarrow{0.99}$ \\
\hline \multirow{3}{*}{$\begin{array}{l}\text { Has your physical health interfered with your ability } \\
\text { to do your daily work at home and/or away from } \\
\text { home? }\end{array}$} & Yes & 78 & $2(8.7)$ & $72(10.2)$ & \\
\hline & No & 676 & $21(91.3)$ & 632(89.4) & \\
\hline & $N A^{*}$ & 86 & $0(0)$ & $3(0.4)$ & $\overrightarrow{0.99}$ \\
\hline \multirow{3}{*}{$\begin{array}{l}\text { During the past month, have you often been } \\
\text { bothered by little interest or pleasure in doing things? }\end{array}$} & Yes & 136 & $4(17.4)$ & 124(17.5) & \\
\hline & No & 619 & 19(82.6) & $581(82.2)$ & \\
\hline & $N A^{*}$ & 85 & $0(0)$ & $2(0.3)$ & $\overrightarrow{0} .99$ \\
\hline \multirow[t]{3}{*}{ The work I do is meaningful to me. } & $\begin{array}{l}\text { Very strongly } \\
\text { disagree }\end{array}$ & 17 & $1(4.3)$ & $16(2.3)$ & \\
\hline & $\begin{array}{l}\text { Strongly } \\
\text { disagree }\end{array}$ & 9 & $1(4.3)$ & $7(1.0)$ & \\
\hline & Disagree & 3 & $0(0)$ & $3(0.4)$ & \\
\hline
\end{tabular}

LGBTQ = Lesbian, Gay, Bisexual, Transgender, and Questioning; NA = Not answered

*Not included in the calculation of the p-value from the Fisher's exact test. 


\begin{tabular}{|c|c|c|c|c|c|}
\hline & & N & $\begin{array}{l}\text { LGBTQ } \\
\text { N(\%) }\end{array}$ & $\begin{array}{l}\text { Straight } \\
\mathrm{N}(\%)\end{array}$ & $\begin{array}{l}\mathrm{P} \\
\text { value }\end{array}$ \\
\hline & Neutral & 34 & $1(4.3)$ & $31(4.4)$ & \\
\hline & Agree & 194 & 7(30.4) & $175(24.8)$ & \\
\hline & $\begin{array}{l}\text { Strongly } \\
\text { agree }\end{array}$ & 298 & $7(30.4)$ & $283(40.0)$ & \\
\hline & $\begin{array}{l}\text { Very Strongly } \\
\text { agree }\end{array}$ & 201 & $6(26.1)$ & $191(27.0)$ & \\
\hline & $N A^{*}$ & 84 & $0(0)$ & $1(0.1)$ & 0.41 \\
\hline \multirow[t]{7}{*}{$\begin{array}{l}\text { How important is your mental health and wellness to } \\
\text { your program? }\end{array}$} & $\begin{array}{l}\text { Very } \\
\text { important }\end{array}$ & 373 & $14(60.9)$ & $350(49.5)$ & \\
\hline & $\begin{array}{l}\text { Moderately } \\
\text { important }\end{array}$ & 214 & $4(17.4)$ & 204(28.9) & \\
\hline & $\begin{array}{l}\text { Somewhat } \\
\text { important }\end{array}$ & 113 & $4(17.4)$ & 104(14.7) & \\
\hline & $\begin{array}{l}\text { Less } \\
\text { important }\end{array}$ & 23 & $0(0)$ & $20(2.8)$ & \\
\hline & $\begin{array}{l}\text { Not important } \\
\text { at all }\end{array}$ & 15 & $1(4.3)$ & $12(1.7)$ & \\
\hline & I'm not sure & 18 & $0(0)$ & $17(2.4)$ & \\
\hline & $N A^{*}$ & 84 & $0(0)$ & $0(0)$ & 0.54 \\
\hline \multicolumn{6}{|l|}{ Satisfaction with Training Program } \\
\hline \multirow{3}{*}{$\begin{array}{l}\text { Are you satisfied with the quality of training you are } \\
\text { receiving? }\end{array}$} & Yes & 709 & $20(87)$ & 677(95.8) & \\
\hline & No & 32 & $3(13)$ & $28(4.0)$ & \\
\hline & $N A^{*}$ & 99 & $0(0)$ & $2(0.3)$ & 0.07 \\
\hline \multirow[t]{6}{*}{ I would recommend my training program to others. } & $\begin{array}{l}\text { Strongly } \\
\text { disagree }\end{array}$ & 19 & $0(0)$ & $17(2.4)$ & \\
\hline & $\begin{array}{l}\text { Moderately } \\
\text { disagree }\end{array}$ & 10 & $0(0)$ & $9(1.3)$ & \\
\hline & $\begin{array}{l}\text { Slightly } \\
\text { disagree }\end{array}$ & 14 & $0(0)$ & $14(2.0)$ & \\
\hline & Slightly agree & 62 & $3(13.0)$ & $58(8.2)$ & \\
\hline & $\begin{array}{l}\text { Moderately } \\
\text { agree }\end{array}$ & 149 & $3(13.0)$ & $143(20.2)$ & \\
\hline & $\begin{array}{l}\text { Strongly } \\
\text { agree }\end{array}$ & 489 & 17(73.9) & $466(65.9)$ & \\
\hline \multicolumn{6}{|c|}{ LGBTQ = Lesbian, Gay, Bisexual, Transgender, and Questioning; NA = Not answered } \\
\hline
\end{tabular}




\begin{tabular}{|l|llll|}
\hline & $\mathbf{N}$ & $\begin{array}{l}\text { LGBTQ } \\
\mathbf{N}(\%)\end{array}$ & $\begin{array}{l}\text { Straight } \\
\mathbf{N}(\%)\end{array}$ & $\begin{array}{l}\mathbf{P} \\
\text { value }\end{array}$ \\
\hline LGBTQ = Lesbian, Gay, Bisexual, Transgender, and Questioning; NA = Not answered & & & \\
\hline NA* & 97 & $0(0)$ & $0(0)$ & 0.83 \\
\hline *Not included in the calculation of the p-value from the Fisher's exact test. & & \\
\hline
\end{tabular}

Survey responses based on race were obtained (Table 4). Regarding professionalism, we found a difference between racial groups in reports of being denied opportunities for training or rewards based on gender $(2.3 \%$ White, 9.3\% Black, 2.7\% Asian, 5.6\% Other; $p=0.02)$ and race ( $0.6 \%$ White, 3.1\% Black, 3.6\% Asian, $7 \%$ Other; $p<0.01)$, and witnessing verbal abuse or joking (2.9\% White, 9.3\% Black, $6.4 \%$ Asian, $8.4 \%$ Other; $p=0.01)$. Regarding satisfaction with their quality of training based on race, trainees self-identifying as Other were more likely to report dissatisfaction with their quality of training $(2.7 \%$ White, $6.2 \%$ Black, $4.6 \%$ Asian, $16 \%$ Other; $p<0.01)$. In regard to well-being, no evidence of difference was identified based on race. After collapsing variables to bimodal responses, we found statistical significance in respondents reporting being publicly humiliated $(p=0.02)$; being subjected to racially or ethnically offensive remarks/names $(p<0.01)$; importance of mental health and wellness to your program $(p=0.01)$; and would [they] recommend their training program to others $(p=0.01)$. We did not find any further significance in respondents reporting being denied opportunities for training or rewards based on gender ( $p$ $=0.06$ ). We found no evidence of differences in experiences among LGBTQ trainees based on race (data not shown). 
Table 4

Analysis of responses to questions regarding professionalism, well-being, and satisfaction with training program based on race.

\begin{tabular}{|c|c|c|c|c|c|c|c|}
\hline & & $\mathbf{N}$ & $\begin{array}{l}\text { White } \\
\mathrm{N}(\%)\end{array}$ & $\begin{array}{l}\text { Black } \\
\text { N(\%) }\end{array}$ & $\begin{array}{l}\text { Asian } \\
\mathbf{N}(\%)\end{array}$ & $\begin{array}{l}\text { Other } \\
\mathrm{N}(\%)\end{array}$ & $\begin{array}{l}P \\
\text { value }\end{array}$ \\
\hline \multicolumn{8}{|l|}{ Professionalism } \\
\hline \multirow[t]{5}{*}{ Been publicly humiliated? } & Never & 691 & 470(89.9) & $31(96.9)$ & $97(89.0)$ & $56(78.9)$ & \\
\hline & Once & 46 & $34(6.5)$ & $0(0)$ & $5(4.6)$ & $5(7.0)$ & \\
\hline & Occasionally & 43 & $15(2.9)$ & $1(3.1)$ & $4(3.7)$ & $9(12.7)$ & \\
\hline & Frequently & 5 & $1(0.2)$ & $0(0)$ & $2(1.8)$ & $1(1.4)$ & \\
\hline & $N A^{*}$ & 55 & $3(0.6)$ & $0(0)$ & $1(0.9)$ & $0(0)$ & $\mathrm{N} / \mathrm{A}$ \\
\hline \multirow{5}{*}{$\begin{array}{l}\text { Been threatened with } \\
\text { physical harm? }\end{array}$} & Never & 763 & $507(96.9)$ & $32(100)$ & 105(96.3) & $68(95.8)$ & \\
\hline & Once & 12 & $8(1.5)$ & $0(0)$ & $3(2.8)$ & $1(1.4)$ & \\
\hline & Occasionally & 8 & $4(0.8)$ & $0(0)$ & $0(0)$ & $2(2.8)$ & \\
\hline & Frequently* & 0 & $0(0)$ & $0(0)$ & $0(0)$ & $0(0)$ & \\
\hline & $N A^{*}$ & 57 & $4(0.8)$ & $0(0)$ & $1(0.9)$ & $0(0)$ & 0.46 \\
\hline \multirow[t]{5}{*}{ Been physically harmed? } & Never & 778 & $515(98.5)$ & $32(100)$ & 107(98.2) & 71(100) & \\
\hline & Once & 6 & $4(0.8)$ & $0(0)$ & $1(0.9)$ & $0(0)$ & \\
\hline & Occasionally* & 0 & $0(0)$ & $0(0)$ & $0(0)$ & $0(0)$ & \\
\hline & Frequently* & 0 & $0(0)$ & $0(0)$ & $0(0)$ & $0(0)$ & \\
\hline & $N A^{*}$ & 56 & $4(0.8)$ & $0(0)$ & $1(0.9)$ & $0(0)$ & $\begin{array}{l}> \\
0.99\end{array}$ \\
\hline \multirow{5}{*}{$\begin{array}{l}\text { Been subjected to } \\
\text { unwanted sexual } \\
\text { advances? }\end{array}$} & Never & 758 & $504(96.4)$ & $31(96.9)$ & 105(96.3) & $68(95.8)$ & \\
\hline & Once & 15 & $9(1.7)$ & $0(0)$ & $2(1.8)$ & $1(1.4)$ & \\
\hline & Occasionally & 11 & $6(1.1)$ & $1(3.1)$ & $1(0.9)$ & $2(2.8)$ & \\
\hline & Frequently* & 0 & $0(0)$ & $0(0)$ & $0(0)$ & $0(0)$ & \\
\hline & $N A^{*}$ & 56 & $4(0.8)$ & $0(0)$ & $1(0.9)$ & $0(0)$ & 0.67 \\
\hline \multirow{3}{*}{$\begin{array}{l}\text { Been subjected to offensive } \\
\text { sexist remarks/names? }\end{array}$} & Never & 712 & $479(91.6)$ & $30(93.8)$ & $98(89.9)$ & $62(87.3)$ & \\
\hline & Once & 26 & $14(2.7)$ & $1(3.1)$ & $4(3.7)$ & $3(4.2)$ & \\
\hline & Occasionally & 43 & $25(4.8)$ & $0(0)$ & $6(5.5)$ & $6(8.5)$ & \\
\hline
\end{tabular}

NA $=$ Not answered

*Not included in the calculation of the p-value from the Fisher's exact test. 


\begin{tabular}{|c|c|c|c|c|c|c|c|}
\hline & & $\mathbf{N}$ & $\begin{array}{l}\text { White } \\
\mathrm{N}(\%)\end{array}$ & $\begin{array}{l}\text { Black } \\
\mathrm{N}(\%)\end{array}$ & $\begin{array}{l}\text { Asian } \\
\mathrm{N}(\%)\end{array}$ & $\begin{array}{l}\text { Other } \\
\mathrm{N}(\%)\end{array}$ & $\begin{array}{l}P \\
\text { value }\end{array}$ \\
\hline & Frequently & 3 & $2(0.4)$ & $0(0)$ & $0(0)$ & $0(0)$ & \\
\hline & $N A^{*}$ & 56 & $3(0.6)$ & $1(3.1)$ & $1(0.9)$ & $0(0)$ & $\mathrm{N} / \mathrm{A}$ \\
\hline \multirow{5}{*}{$\begin{array}{l}\text { Been denied opportunities } \\
\text { for training or rewards } \\
\text { based on gender? }\end{array}$} & Never & 756 & $507(96.9)$ & $29(90.6)$ & 105(96.3) & $67(94.4)$ & \\
\hline & Once & 12 & $8(1.5)$ & $2(6.2)$ & $0(0)$ & $1(1.4)$ & \\
\hline & Occasionally & 13 & $3(0.6)$ & $1(3.1)$ & $2(1.8)$ & $3(4.2)$ & \\
\hline & Frequently & 3 & $1(0.2)$ & $0(0)$ & $1(0.9)$ & $0(0)$ & \\
\hline & $N A^{*}$ & 56 & $4(0.8)$ & $0(0)$ & $1(0.9)$ & $0(0)$ & 0.02 \\
\hline \multirow{5}{*}{$\begin{array}{l}\text { Been subjected racially or } \\
\text { ethnically offensive } \\
\text { remarks/names? }\end{array}$} & Never & 718 & $500(95.6)$ & $29(90.6)$ & $86(78.9)$ & $59(83.1)$ & \\
\hline & Once & 30 & $8(1.5)$ & $1(3.1)$ & $12(11.0)$ & $5(7.0)$ & \\
\hline & Occasionally & 33 & 10(1.9) & $2(6.2)$ & $9(8.3)$ & $7(9.9)$ & \\
\hline & Frequently & 1 & $0(0)$ & $0(0)$ & $1(0.9)$ & $0(0)$ & \\
\hline & $N A^{*}$ & 58 & $5(1.0)$ & $0(0)$ & $1(0.9)$ & $0(0)$ & $\mathrm{N} / \mathrm{A}$ \\
\hline \multirow{5}{*}{$\begin{array}{l}\text { Been denies opportunities } \\
\text { for training or rewards } \\
\text { based on race or ethnicity? }\end{array}$} & Never & 766 & 515(98.5) & $29(90.6)$ & $104(95.4)$ & 66(93.0) & \\
\hline & Once & 7 & $3(0.6)$ & $0(0)$ & $1(0.9)$ & $2(2.8)$ & \\
\hline & Occasionally & 6 & $0(0)$ & $1(3.1)$ & $2(1.8)$ & $3(4.2)$ & \\
\hline & Frequently & 1 & $0(0)$ & $0(0)$ & $1(0.9)$ & $0(0)$ & \\
\hline & $N A^{*}$ & 60 & $5(1.0)$ & $2(6.2)$ & $1(0.9)$ & $0(0)$ & $\begin{array}{l}< \\
0.01\end{array}$ \\
\hline \multirow{5}{*}{$\begin{array}{l}\text { Been subjected to offensive } \\
\text { remarks/names related to } \\
\text { sexual orientation? }\end{array}$} & Never & 775 & $517(98.9)$ & $31(96.9)$ & 107(98.2) & 70(98.6) & \\
\hline & Once & 4 & $2(0.4)$ & $0(0)$ & $0(0)$ & $1(1.4)$ & \\
\hline & Occasionally & 3 & $1(0.2)$ & $0(0)$ & $1(0.9)$ & $0(0)$ & \\
\hline & Frequently & 1 & $0(0)$ & $0(0)$ & $0(0)$ & $0(0)$ & \\
\hline & $N A^{*}$ & 57 & $3(0.6)$ & 1(3.1) & $1(0.9)$ & $0(0)$ & 0.42 \\
\hline
\end{tabular}

$\mathrm{NA}=$ Not answered

*Not included in the calculation of the p-value from the Fisher's exact test. 


\begin{tabular}{|c|c|c|c|c|c|c|c|}
\hline & & $\mathbf{N}$ & $\begin{array}{l}\text { White } \\
\mathrm{N}(\%)\end{array}$ & $\begin{array}{l}\text { Black } \\
\mathrm{N}(\%)\end{array}$ & $\begin{array}{l}\text { Asian } \\
\mathrm{N}(\%)\end{array}$ & $\begin{array}{l}\text { Other } \\
\mathrm{N}(\%)\end{array}$ & $\begin{array}{l}P \\
\text { value }\end{array}$ \\
\hline \multirow{5}{*}{$\begin{array}{l}\text { Been denied opportunities } \\
\text { for training or rewards } \\
\text { based on sexual } \\
\text { orientation? }\end{array}$} & Never & 780 & $518(99.0)$ & $31(96.9)$ & 107(98.2) & $71(100)$ & \\
\hline & Once & 2 & $1(0.2)$ & $0(0)$ & $1(0.9)$ & $0(0)$ & \\
\hline & Occasionally* & 0 & $0(0)$ & $0(0)$ & $0(0)$ & $0(0)$ & \\
\hline & Frequently* & 0 & $0(0)$ & $0(0)$ & $0(0)$ & $0(0)$ & \\
\hline & $N A^{*}$ & 58 & $4(0.8)$ & $1(3.1)$ & $1(0.9)$ & $0(0)$ & 0.49 \\
\hline \multirow{5}{*}{$\begin{array}{l}\text { Shouting, use of belittling } \\
\text { or abusive language }\end{array}$} & Never & 632 & $429(82.0)$ & $29(90.6)$ & $90(82.6)$ & $56(78.9)$ & \\
\hline & Once & 63 & $46(8.8)$ & $2(6.2)$ & $8(7.3)$ & $4(5.6)$ & \\
\hline & Occasionally & 70 & $44(8.4)$ & $1(3.1)$ & $8(7.3)$ & $7(9.9)$ & \\
\hline & Frequently & 6 & $1(0.2)$ & $0(0)$ & $2(1.8)$ & $2(2.8)$ & \\
\hline & $N A^{*}$ & 69 & $3(0.6)$ & $0(0)$ & $1(0.9)$ & $2(2.8)$ & $\mathrm{N} / \mathrm{A}$ \\
\hline \multirow{5}{*}{$\begin{array}{l}\text { Verbal, written, or physical } \\
\text { personal attacks directed at } \\
\text { others }\end{array}$} & Never & 705 & $485(92.7)$ & $29(90.6)$ & $99(90.8)$ & $62(87.3)$ & \\
\hline & Once & 27 & 16(3.1) & $2(6.2)$ & $4(3.7)$ & $1(1.4)$ & \\
\hline & Occasionally & 36 & $20(3.8)$ & 1(3.1) & $4(3.7)$ & $5(7.0)$ & \\
\hline & Frequently & 4 & $0(0)$ & $0(0)$ & $1(0.9)$ & $2(2.8)$ & \\
\hline & $N A^{*}$ & 68 & $2(0.4)$ & $0(0)$ & $1(0.9)$ & $1(1.4)$ & $\mathrm{N} / \mathrm{A}$ \\
\hline \multirow{5}{*}{$\begin{array}{l}\text { Public derogatory } \\
\text { comments about quality of } \\
\text { care by other professionals }\end{array}$} & Never & 631 & $433(82.8)$ & $29(90.6)$ & $90(82.6)$ & $56(78.9)$ & \\
\hline & Once & 34 & $20(3.8)$ & $0(0)$ & $7(6.4)$ & $5(7.0)$ & \\
\hline & Occasionally & 104 & $68(13.0)$ & $3(9.4)$ & $10(9.2)$ & $8(11.3)$ & \\
\hline & Frequently & 4 & $1(0.2)$ & $0(0)$ & $1(0.9)$ & $1(1.4)$ & \\
\hline & $N A^{*}$ & 67 & $1(0.2)$ & $0(0)$ & $1(0.9)$ & 1(1.4) & $\mathrm{N} / \mathrm{A}$ \\
\hline \multirow{3}{*}{$\begin{array}{l}\text { Inappropriate expressions } \\
\text { of anger (e.g., throwing } \\
\text { things, destruction of } \\
\text { property) }\end{array}$} & Never & 738 & $501(95.8)$ & $31(96.9)$ & $103(94.5)$ & $66(93.0)$ & \\
\hline & Once & 18 & $11(2.1)$ & $1(3.1)$ & $2(1.8)$ & $2(2.8)$ & \\
\hline & Occasionally & 11 & $6(1.1)$ & $0(0)$ & $2(1.8)$ & $1(1.4)$ & \\
\hline \multicolumn{8}{|l|}{ NA $=$ Not answered } \\
\hline
\end{tabular}




\begin{tabular}{|c|c|c|c|c|c|c|c|}
\hline & & $\mathbf{N}$ & $\begin{array}{l}\text { White } \\
\mathrm{N}(\%)\end{array}$ & $\begin{array}{l}\text { Black } \\
\mathrm{N}(\%)\end{array}$ & $\begin{array}{l}\text { Asian } \\
\mathrm{N}(\%)\end{array}$ & $\begin{array}{l}\text { Other } \\
\mathrm{N}(\%)\end{array}$ & $\begin{array}{l}\mathrm{P} \\
\text { value }\end{array}$ \\
\hline & Frequently & 2 & $0(0)$ & $0(0)$ & $1(0.9)$ & $1(1.4)$ & \\
\hline & $N A^{\star}$ & 71 & $5(1.0)$ & $0(0)$ & $1(0.9)$ & 1(1.4) & 0.32 \\
\hline \multirow[t]{5}{*}{ Physical assault } & Never & 770 & $521(99.6)$ & $31(96.9)$ & 108(99.1) & 69(97.2) & \\
\hline & Once & 1 & $0(0)$ & $0(0)$ & $0(0)$ & $1(1.4)$ & \\
\hline & Occasionally* & 0 & $0(0)$ & $0(0)$ & $0(0)$ & $0(0)$ & \\
\hline & Frequently* & 0 & $0(0)$ & $0(0)$ & $0(0)$ & $0(0)$ & \\
\hline & $N A^{*}$ & 69 & $2(0.4)$ & $1(3.1)$ & $1(0.9)$ & $1(1.4)$ & 0.14 \\
\hline \multirow{5}{*}{$\begin{array}{l}\text { Unwelcome or wanted } \\
\text { physical advances of a } \\
\text { sexual nature }\end{array}$} & Never & 762 & $516(98.7)$ & $31(96.9)$ & 106(97.2) & $68(95.8)$ & \\
\hline & Once & 4 & $2(0.4)$ & $0(0)$ & $1(0.9)$ & $1(1.4)$ & \\
\hline & Occasionally & 5 & $2(0.4)$ & $1(3.1)$ & $1(0.9)$ & $1(1.4)$ & \\
\hline & Frequently* & 0 & $0(0)$ & $0(0)$ & $0(0)$ & $0(0)$ & \\
\hline & $N A^{*}$ & 69 & $3(0.6)$ & $0(0)$ & $1(0.9)$ & $1(1.4)$ & 0.14 \\
\hline \multirow{5}{*}{$\begin{array}{l}\text { Verbal abuse or joking } \\
\text { based on gender }\end{array}$} & Never & 712 & 488(93.3) & $29(90.6)$ & 103(94.5) & $60(84.5)$ & \\
\hline & Once & 20 & $12(2.3)$ & $1(3.1)$ & $3(2.8)$ & $3(4.2)$ & \\
\hline & Occasionally & 36 & 20(3.8) & $2(6.2)$ & $1(0.9)$ & $5(7.0)$ & \\
\hline & Frequently & 1 & $0(0)$ & $0(0)$ & $1(0.9)$ & $0(0)$ & \\
\hline & $N A^{*}$ & 71 & $3(0.6)$ & $0(0)$ & $1(0.9)$ & $3(4.2)$ & 0.21 \\
\hline \multirow{5}{*}{$\begin{array}{l}\text { Verbal abuse or joking } \\
\text { based on race/ethnicity }\end{array}$} & Never & 735 & $505(96.6)$ & $29(90.6)$ & 101(92.7) & 63(88.7) & \\
\hline & Once & 13 & $5(1.0)$ & $1(3.1)$ & $5(4.6)$ & $2(2.8)$ & \\
\hline & Occasionally & 21 & 10(1.9) & $2(6.2)$ & $1(0.9)$ & $4(5.6)$ & \\
\hline & Frequently & 1 & $0(0)$ & $0(0)$ & $1(0.9)$ & $0(0)$ & \\
\hline & $N A^{*}$ & 70 & $3(0.6)$ & $0(0)$ & $1(0.9)$ & $2(2.8)$ & 0.01 \\
\hline \multirow{4}{*}{$\begin{array}{l}\text { Verbal abuse or joking } \\
\text { based on sexual orientation }\end{array}$} & Never & 754 & $511(97.7)$ & $32(100)$ & 105(96.3) & $68(95.8)$ & \\
\hline & Once & 7 & $4(0.8)$ & $0(0)$ & $3(2.8)$ & $0(0)$ & \\
\hline & Occasionally & 9 & $5(1.0)$ & $0(0)$ & $0(0)$ & $1(1.4)$ & \\
\hline & Frequently* & 0 & $0(0)$ & $0(0)$ & $0(0)$ & $0(0)$ & \\
\hline \multicolumn{8}{|l|}{$\mathrm{NA}=$ Not answered } \\
\hline
\end{tabular}




\begin{tabular}{|c|c|c|c|c|c|c|c|}
\hline & & $\mathbf{N}$ & $\begin{array}{l}\text { White } \\
\mathrm{N}(\%)\end{array}$ & $\begin{array}{l}\text { Black } \\
\mathrm{N}(\%)\end{array}$ & $\begin{array}{l}\text { Asian } \\
\mathrm{N}(\%)\end{array}$ & $\begin{array}{l}\text { Other } \\
\mathrm{N}(\%)\end{array}$ & $\begin{array}{l}P \\
\text { value }\end{array}$ \\
\hline & $N A^{*}$ & 70 & $3(0.6)$ & $0(0)$ & $1(0.9)$ & $2(2.8)$ & 0.41 \\
\hline \multicolumn{8}{|l|}{ Well-Being } \\
\hline \multirow{3}{*}{$\begin{array}{l}\text { Have you felt emotionally } \\
\text { drained from your work? }\end{array}$} & Yes & 424 & $306(58.5)$ & 15(46.9) & $52(47.7)$ & $37(52.1)$ & \\
\hline & No & 331 & $216(41.3)$ & $17(53.1)$ & $56(51.4)$ & $34(47.9)$ & \\
\hline & $N A^{*}$ & 85 & $1(0.2)$ & $0(0)$ & $1(0.9)$ & $0(0)$ & 0.13 \\
\hline \multirow{3}{*}{$\begin{array}{l}\text { Have you worried that your } \\
\text { work is hardening you } \\
\text { emotionally? }\end{array}$} & Yes & 329 & $238(45.5)$ & $10(31.2)$ & $41(37.6)$ & $31(43.7)$ & \\
\hline & No & 428 & $285(54.5)$ & $22(68.8)$ & $68(62.4)$ & $40(56.3)$ & \\
\hline & $N A^{*}$ & 83 & $0(0)$ & $0(0)$ & $0(0)$ & $0(0)$ & 0.23 \\
\hline \multirow{3}{*}{$\begin{array}{l}\text { Have you often been } \\
\text { bothered by feeling down, } \\
\text { depressed, or hopeless? }\end{array}$} & Yes & 189 & $127(24.3)$ & $9(28.1)$ & $28(25.7)$ & $19(26.8)$ & \\
\hline & No & 565 & $395(75.5)$ & 23(71.9) & $80(73.4)$ & $51(71.8)$ & \\
\hline & $N A^{\star}$ & 86 & $1(0.2)$ & $0(0)$ & $1(0.9)$ & 1(1.4) & 0.88 \\
\hline \multirow{3}{*}{$\begin{array}{l}\text { Have you fallen asleep } \\
\text { while sitting inactive in a } \\
\text { public place? }\end{array}$} & Yes & 138 & $94(18)$ & $5(15.6)$ & 17(15.6) & $17(23.9)$ & \\
\hline & No & 618 & 429(82) & $27(84.4)$ & $91(83.5)$ & $54(76.1)$ & \\
\hline & $N A^{*}$ & 84 & $0(0)$ & $0(0)$ & $1(0.9)$ & $0(0)$ & 0.55 \\
\hline \multirow{3}{*}{$\begin{array}{l}\text { Have you felt that all things } \\
\text { you had to do were piling } \\
\text { up so high that you could } \\
\text { not overcome them? }\end{array}$} & Yes & 210 & $147(28.1)$ & $10(31.2)$ & $31(28.4)$ & $18(25.4)$ & \\
\hline & No & 545 & $376(71.9)$ & $22(68.8)$ & $77(70.6)$ & $52(73.2)$ & \\
\hline & $N A^{*}$ & 85 & $0(0)$ & $0(0)$ & $1(0.9)$ & 1(1.4) & 0.94 \\
\hline \multirow{3}{*}{$\begin{array}{l}\text { Have you been bothered by } \\
\text { emotional problems (such } \\
\text { as feeling anxious, } \\
\text { depressed, or irritable)? }\end{array}$} & Yes & 312 & $214(40.9)$ & $13(40.6)$ & $45(41.3)$ & $34(47.9)$ & \\
\hline & No & 442 & $306(58.5)$ & 19(59.4) & 64(58.7) & $37(52.1)$ & \\
\hline & $N A^{*}$ & 86 & $3(0.6)$ & $0(0)$ & $0(0)$ & $0(0)$ & 0.75 \\
\hline
\end{tabular}

$\mathrm{NA}=$ Not answered

*Not included in the calculation of the p-value from the Fisher's exact test. 


\begin{tabular}{|c|c|c|c|c|c|c|c|}
\hline & & $\mathbf{N}$ & $\begin{array}{l}\text { White } \\
\mathrm{N}(\%)\end{array}$ & $\begin{array}{l}\text { Black } \\
\mathrm{N}(\%)\end{array}$ & $\begin{array}{l}\text { Asian } \\
\mathrm{N}(\%)\end{array}$ & $\begin{array}{l}\text { Other } \\
\mathrm{N}(\%)\end{array}$ & $\begin{array}{l}P \\
\text { value }\end{array}$ \\
\hline \multirow{3}{*}{$\begin{array}{l}\text { Has your physical health } \\
\text { interfered with your ability } \\
\text { to do your daily work at } \\
\text { home and/or away from } \\
\text { home? }\end{array}$} & Yes & 78 & $48(9.2)$ & $5(15.6)$ & 11(10.1) & $10(14.1)$ & \\
\hline & No & 676 & $473(90.4)$ & $27(84.4)$ & $98(89.9)$ & $60(84.5)$ & \\
\hline & $N A^{*}$ & 86 & $2(0.4)$ & $0(0)$ & $0(0)$ & $1(1.4)$ & 0.34 \\
\hline \multirow{3}{*}{$\begin{array}{l}\text { During the past month, } \\
\text { have you often been } \\
\text { bothered by little interest or } \\
\text { pleasure in doing things? }\end{array}$} & Yes & 136 & $94(18.0)$ & $7(21.9)$ & 15(13.8) & 14(19.7) & \\
\hline & No & 619 & $428(81.8)$ & $25(78.1)$ & $94(86.4)$ & $56(78.9)$ & \\
\hline & $N A^{*}$ & 85 & $1(0.2)$ & $0(0)$ & $0(0)$ & $1(1.4)$ & 0.57 \\
\hline \multirow[t]{8}{*}{$\begin{array}{l}\text { The work I do is meaningful } \\
\text { to me. }\end{array}$} & $\begin{array}{l}\text { Very strongly } \\
\text { disagree }\end{array}$ & 17 & $9(1.7)$ & $1(3.1)$ & $5(4.6)$ & $2(2.8)$ & \\
\hline & $\begin{array}{l}\text { Strongly } \\
\text { disagree }\end{array}$ & 9 & $5(1.0)$ & $1(3.1)$ & $2(1.8)$ & $0(0)$ & \\
\hline & Disagree & 3 & $2(0.4)$ & $0(0)$ & $0(0)$ & $1(1.4)$ & \\
\hline & Neutral & 34 & $22(4.2)$ & $0(0)$ & $6(5.5)$ & $5(7.0)$ & \\
\hline & Agree & 194 & $141(27.0)$ & $4(12.5)$ & $27(24.8)$ & $14(19.7)$ & \\
\hline & $\begin{array}{l}\text { Strongly } \\
\text { Agree }\end{array}$ & 298 & $209(40.0)$ & $15(46.9)$ & $44(40.4)$ & $23(32.4)$ & \\
\hline & $\begin{array}{l}\text { Very Strongly } \\
\text { Agree }\end{array}$ & 201 & $135(25.8)$ & 11(34.4) & $24(22.0)$ & $26(36.6)$ & \\
\hline & $N A^{*}$ & 84 & $0(0)$ & $0(0)$ & $1(0.9)$ & $0(0)$ & $\mathrm{N} / \mathrm{A}$ \\
\hline \multirow{6}{*}{$\begin{array}{l}\text { How important is your } \\
\text { mental health and wellness } \\
\text { to your program? }\end{array}$} & $\begin{array}{l}\text { Very } \\
\text { important }\end{array}$ & 373 & 258(49.3) & $18(56.2)$ & $50(45.9)$ & 39(54.9) & \\
\hline & $\begin{array}{l}\text { Moderately } \\
\text { important }\end{array}$ & 214 & $160(30.6)$ & $7(21.9)$ & $30(27.5)$ & $13(18.3)$ & \\
\hline & $\begin{array}{l}\text { Somewhat } \\
\text { important }\end{array}$ & 113 & $77(14.7)$ & $5(15.6)$ & 18(16.5) & $8(11.3)$ & \\
\hline & $\begin{array}{l}\text { Less } \\
\text { important }\end{array}$ & 23 & $12(2.3)$ & $0(0)$ & $3(2.8)$ & $6(8.5)$ & \\
\hline & $\begin{array}{l}\text { Not important } \\
\text { at all }\end{array}$ & 15 & $5(1.0)$ & $1(3.1)$ & $4(3.7)$ & $4(5.6)$ & \\
\hline & I'm not sure & 18 & $11(2.1)$ & $1(3.1)$ & $4(3.7)$ & $1(1.4)$ & \\
\hline \multicolumn{8}{|l|}{$\mathrm{NA}=$ Not answered } \\
\hline
\end{tabular}




\begin{tabular}{|c|c|c|c|c|c|c|c|}
\hline & & $\mathbf{N}$ & $\begin{array}{l}\text { White } \\
\mathrm{N}(\%)\end{array}$ & $\begin{array}{l}\text { Black } \\
\mathrm{N}(\%)\end{array}$ & $\begin{array}{l}\text { Asian } \\
\mathrm{N}(\%)\end{array}$ & $\begin{array}{l}\text { Other } \\
\mathrm{N}(\%)\end{array}$ & $\begin{array}{l}P \\
\text { value }\end{array}$ \\
\hline & $N A^{*}$ & 84 & $0(0)$ & $0(0)$ & $0(0)$ & $0(0)$ & $\mathrm{N} / \mathrm{A}$ \\
\hline \multicolumn{8}{|c|}{ Satisfaction with Training Program } \\
\hline \multirow{3}{*}{$\begin{array}{l}\text { Are you satisfied with the } \\
\text { quality of training you are } \\
\text { receiving? }\end{array}$} & Yes & 709 & $507(96.9)$ & $30(93.8)$ & $104(95.4$ & $60(84.5)$ & \\
\hline & No & 32 & $14(2.7)$ & $2(6.2)$ & $5(4.6)$ & $11(15.5)$ & \\
\hline & $N A^{*}$ & 99 & $2(0.4)$ & $0(0)$ & $0(0)$ & $0(0)$ & $\begin{array}{l}< \\
0.01\end{array}$ \\
\hline \multirow[t]{7}{*}{$\begin{array}{l}\text { I would recommend my } \\
\text { training program to others. }\end{array}$} & $\begin{array}{l}\text { Strongly } \\
\text { disagree }\end{array}$ & 19 & $9(1.7)$ & $0(0)$ & $7(6.4)$ & $2(2.8)$ & \\
\hline & $\begin{array}{l}\text { Moderately } \\
\text { disagree }\end{array}$ & 10 & $4(0.8)$ & $3(9.4)$ & $2(1.8)$ & $1(1.4)$ & \\
\hline & $\begin{array}{l}\text { Slightly } \\
\text { disagree }\end{array}$ & 14 & $8(1.5)$ & $1(3.1)$ & $1(0.9)$ & $4(5.6)$ & \\
\hline & Slightly agree & 62 & $34(6.5)$ & $3(9.4)$ & $12(11.0)$ & $11(15.5)$ & \\
\hline & $\begin{array}{l}\text { Moderately } \\
\text { agree }\end{array}$ & 149 & 103(19.7) & $4(12.5)$ & $26(23.6)$ & $15(21.1)$ & \\
\hline & $\begin{array}{l}\text { Strongly } \\
\text { agree }\end{array}$ & 489 & $365(69.8)$ & $21(65.6)$ & $61(56.0)$ & $38(53.5)$ & \\
\hline & $N A^{*}$ & 97 & $0(0)$ & $0(0)$ & $0(0)$ & $0(0)$ & $\mathrm{N} / \mathrm{A}$ \\
\hline \multicolumn{8}{|l|}{$N A=$ Not answered } \\
\hline
\end{tabular}

\section{Discussion}

This is the first study to assess the training experiences of LGBTQ trainees in a southern training program utilizing a well-being framework (PERMA). To our knowledge, this is the only study that solely compared LGBTQ and Straight residents and fellows. Three percent of trainees identify as LGBTQ, consistent with the percent of LGBTQ residents in Alabama and the country as a whole. 3,8 We also identified variability in experiences regarding professionalism/relationships between LGBTQ and Straight trainees at a single, large teaching institution, with some of the variability explained by intersectionalities such as race.

\section{Assessment of well-being (Positive emotion \& Meaning)}

Our study found no evidence of differences in well-being/positive emotion between LGBTQ and Straight trainees. In addition, over $60 \%$ of respondents felt the institution and their respective department were LGBTQ friendly; however, it is unclear how many trainees identifying as LGBTQ shared this sentiment. Understanding LGBTQ trainees' perspectives of their department's environment is important because literature has found LGBTQ individuals are at an increased risk for problems with mental health (e.g., depression, suicide, psychosocial disorders). ${ }^{13-19}$ Also, 
having a supportive environment fosters more social interaction and allows LGBTQ individuals the ability to create bonds and potential mentorship. ${ }^{20,21}$

\section{Fear of disclosure (Relationships)}

LBTQ stigma in "The South" continues to be prominent and still plagues the minds of trainees considering moving to these areas. The fear of sexual orientation disclosure is a common concern among LGBTQ trainees and interferes with formation of relationships. Prior studies identify LGBTQ trainees do not desire to disclose their sexual orientation due to fear of harassment, discrimination, and missed career opportunities. ${ }^{5,22-26}$ We did not identify any differences between LGBTQ and Straight trainees pertaining to being denied opportunities based on sexual orientation $(p=0.06)$.

\section{Barriers in the workplace (Engagement \& Accomplishment)}

Lastly, institutions are only beginning to identify barriers to LGBTQ trainees' experiences in the workplace. Most participants felt the institution and their department were LGBTQ friendly; however, we are unclear if this same perspective is shared by LGBTQ trainees specifically. Eliason, et al. found that LGBTQ physicians reported being denied referrals from heterosexual/Straight colleagues and were denied privileges or promotion based on their sexuality. ${ }^{22}$ Some studies have also shown that LGBTQ medical students, trainees, and physicians are likely to witness derogatory comments, substandard care, or refusal of care towards LGBTQ patients, which was also seen in our cohort. ${ }^{22,27}$ These barriers impede trainees' abilities to fully engage in their work and achieve a sense of accomplishment, thereby inhibiting their ability to flourish in their environment. This is best illustrated by our study finding only $0.1 \%$ of trainees who identified as LGBTQ were willing to mentor LGBTQ individuals, suggesting individuals may not be able to dedicate themselves to something or someone for the greater good.

\section{Intersectionalities (Positive Emotion, Engagement, and Accomplishment)}

Our study also suggested intersectionality may be important. Ideally, each component of the PERMA theory is assessed individually; however, the complexity of the effects of intersectionality on individuals may impact their positive emotion, engagement, or accomplishment.

Despite the growing acknowledgment by the AAMC and LCME for a racially diverse workforce, there continues to be significant gaps in making training environments inclusive as is evidenced by $71 \%$ of survey respondents being White/Caucasian and $56 \%$ identifying as male. URiM trainees are more likely to care for underrepresented minorities, ${ }^{28,29}$ and are also more likely to experience burnout due to implicit and explicit racial biases compared to their White colleagues. ${ }^{30}$ Furthermore, according to the most recent 2020 AAMC U.S. Medical School Faculty report, Black and Hispanic physicians are less likely to be in faculty positions compared to Whites and Asians. ${ }^{31}$ Although, LGBTQ trainees' experiences did not vary based on race secondary to low rates of self-reported identification as LGBTQ, understanding how LGBTQ and race intersect among trainees is crucial. These experiences, whether positive or negative, can be compounded in individuals who identify with multiple URiM groups (e.g., Black and Gay, Native American and Lesbian, Latino and Transgender, etc.). ${ }^{27}$

\section{Limitations}

This study should be viewed within the context of some limitations. Our cohort is from a single, academic institution within the Southeast and cannot be generalized to other academic centers in other geographic locations. 
The small cohort of LGBTQ trainees, those similar to other academic institutions, limits statistical power and assessment of intersectionalities based on race. Recall bias also may have influenced our findings due to the nature of the study. In addition, 110 (13\%) responders were excluded due to lack of response to sexual orientation.

\section{Future Studies}

Future studies are needed to assess the LGBTQ experience at other large academic training institutions to identify common trends to guide universal changes in GME and provide optimal training environments for all individuals regardless of sexual orientation or background.

\section{Conclusion}

Expanding our knowledge of the current LGBTQ and URiM trainee workforce is imperative. This study is the first to contrast the dynamics of GME training between LGBTQ and Straight trainees and highlight the disparities seen with an integrated PERMA and intersectionality framework. Increasing diversity and inclusivity will not only alleviate suffering but allow LGBTQ trainees to flourish and thus improve health outcomes for LGBTQ residents. It is crucial to continue supporting the advancement of this field of study in order to retain and recruit diverse physicians who are interested in improving the quality of LGBTQ healthcare.

\section{Abbreviations}

AAMC

Association of American Medical Colleges; URiM = Underrepresented in Medicine; LGBTQ = Lesbian, Gay, Bisexual, Transgender, and Questioning; PERMA = Positive emotion, Engagement, Relationships, Meaning, and Accomplishment; UAB = University of Alabama at Birmingham; GME = General Medical Education; PGY = Postgraduate year; ACGME = Accreditation Council of General Medical Education; NA = Not answered

\section{Declarations}

\section{Ethics approval and consent to participate}

This study was approved by the Institutional Review Board at the University of Alabama at Birmingham.

\section{Consent for publication}

All authors have reviewed the manuscript and agree with submission to the journal in its current form.

\section{Availability of data and material}

The data is not available for request.

\section{Competing interests}

The authors declare no conflict of interest.

\section{Funding}

There was no additional funding for this study. 


\section{Authors' Contributions}

Z.W.W. was the principal investigator for the study was responsible for conceptualization of the idea, study design, analysis of data, manuscript writing and editing. M.A. and I.C. were responsible for methodology, data interpretation and analysis. B.M.L. was responsible for study design and manuscript editing. L.E.E. was responsible for study design, data analysis, and manuscript editing. A.R.G. was responsible for manuscript editing. S.V.H. was the supervisor for the study and was responsible for helping Z.W.W. with study design, analysis of data, and manuscript editing.

\section{Acknowledgments}

The authors would like to thank the staff and administration within the UAB GME Department who helped with dispersing the online survey.

\section{References}

1. Liasion Committee on Medical Education Liaison Committee on Medical Education (LCME) standards on diversity.

https://health.usf.edu/ /media/Files/Medicine/MD\%20Program/Diversity/LCMEStandardsonDiversity1.ashx? la=en. Accessed January 8, 2019.

2. Association of American Medical Colleges Underrepresented in medicine definition. https://www.aamc.org/initiatives/urm/. Accessed January 8, 2019.

3. Hafeez H, Zeshan M, Tahir MA, Jahan N, Naveed S. Health Care Disparities Among Lesbian, Gay, Bisexual, and Transgender Youth: A Literature Review. Cureus. 2017;9(4). doi:10.7759/cureus.1184

4. Patridge E V., Barthelemy RS, Rankin SR. Factors impacting the academic climate for LGBQ stem faculty. $J$ Women Minor Sci Eng. Published online 2014. doi:10.1615/JWomenMinorScienEng.2014007429

5. Rankin SR. Campus climates for sexual minorities. New Dir Student Serv. Published online 2005. doi:10.1002/ss.170

6. Mansh M, White W, Gee-Tong L, et al. Sexual and Gender Minority Identity Disclosure during Undergraduate Medical Education: "in the Closet" in Medical School. In: Academic Medicine; 2015. doi:10.1097/ACM.0000000000000657

7. Sanlo R. Lesbian, Gay, and Bisexual College Students: Risk, Resiliency, and Retention. J Coll Student Retent Res Theory Pract. Published online 2004. doi:10.2190/fh61-ve7v-hhcx-0pur

8. LGBT Demographic Data Interactive. (January 2019). Los Angeles, CA: The Williams Institute, UCLA School of Law.

9. “Healthcare Equality Index 2019." HRC, .

10. Seligman M. PERMA and the building blocks of well-being. J Posit Psychol. 2018;13(4):333-335. doi:10.1080/17439760.2018.1437466

11. Dimant OE, Cook TE, Greene RE, Radix AE. Experiences of Transgender and Gender Nonbinary Medical Students and Physicians. In: Transgender Health.; 2019. doi:10.1089/trgh.2019.0021

12. Sánchez NF, Rankin S, Callahan E, et al. LGBT trainee and health professional perspectives on academic careers - Facilitators and challenges. LGBT Heal. Published online 2015. doi:10.1089/lgbt.2015.0024 
13. Mays VM, Cochran SD. Mays and Cochran / Peer Reviewed / Research Articles / 1869 Public Health. Vol 91.; 2001. http://midmac.med.harvard.edu.

14. Meyer IH. Prejudice, Social Stress, and Mental Health in Lesbian, Gay, and Bisexual Populations: Conceptual Issues and Research Evidence. Psychol Bull. 2003;129(5):674-697. doi:10.1037/0033-2909.129.5.674

15. Murdock TB, Bolch MB. Risk and protective factors for poor school adjustment in lesbian, gay, and bisexual (LGB) high school youth: Variable and person-centered analyses. Psychol Sch. 2005;42(2):159-172. doi:10.1002/pits.20054

16. Williams T, Connolly J, Pepler D, Craig W. Peer victimization, social support, and psychosocial adjustment of sexual minority adolescents. J Youth Adolesc. 2005;34(5):471-482. doi:10.1007/s10964-005-7264-x

17. Cochran SD, Keenan C, Schober C, Mays VM. Estimates of alcohol use and clinical treatment needs among homosexually active men and women in the U.S. population. J Consult Clin Psychol. 2000;68(6):1062-1071. doi:10.1037//0022-006x.68.6.1062

18. Drabble L, Trocki K. Alcohol consumption, alcohol-related problems, and other substance use among lesbian and bisexual women. J Lesbian Stud. 2005;9(3):19-30. doi:10.1300/J155v09n03_03

19. Kipke MD, Weiss G, Ramirez M, et al. Club drug use in Los Angeles among young men who have sex with men. Subst Use Misuse. 2007;42(11):1723-1743. doi:10.1080/10826080701212261

20. Townsend $\mathrm{MH}$, Wallick MM, Cambre KM. Follow-up survey of support services for lesbian, gay, and bisexual medical students. Acad Med. 1996;71(9):1012-1014. doi:10.1097/00001888-199609000-00018

21. Lovell B. "We are a tight community": Social groups and social identity in medical undergraduates. Med Educ. 2015;49(10):1016-1027. doi:10.1111/medu.12781

22. Eliason MJ, Dibble SL, Robertson PA. Lesbian, Gay, Bisexual, and Transgender (LGBT) Physicians' Experiences in the Workplace. J Homosex. Published online 2011. doi:10.1080/00918369.2011.614902

23. Eliason MJ, Streed C, Henne M. Coping With Stress as an LGBTQ + Health Care Professional. J Homosex. 2018;65(5):561-578. doi:10.1080/00918369.2017.1328224

24. RF F. State loses gay faculty over benefits policy. Associated Press.

25. Kaminski D, Geisler C. Survival analysis of faculty retention in science and engineering by gender. Science (80-). 2012;335(6070):864-866. doi:10.1126/science.1214844

26. Lapinski J, Sexton P. Still in the closet: The invisible minority in medical education. BMC Med Educ. Published online 2014. doi:10.1186/1472-6920-14-171

27. Toman L. Navigating medical culture and LGBTQ identity. Clin Teach. Published online 2019. doi:10.1111/tct.13078

28. Association of Medical Colleges. Addressing racial disparities in health care: A targeted action plan for academic medical centers. Published 2009. Accessed April 18, 2019.

https://members.aamc.org/eweb/upload/addressing racial disparaties.pdf.

29. Wayne SJ, Kalishman S, Jerabek RN, Timm C, Cosgrove E. Early predictors of physicians' practice in medically underserved communities: A 12-year follow-up study of University of New Mexico School of Medicine graduates. Acad Med. 2010;85(10 suppl):S13-S16.

30. Dyrbye L, Herrin J, West CP, et al. Association of Racial Bias with Burnout among Resident Physicians. JAMA Netw Open. Published online 2019. doi:10.1001/jamanetworkopen.2019.7457

31. 2020 U.S. Medical School Faculty | AAMC. Accessed April 7, 2021. https://www.aamc.org/data-reports/facultyinstitutions/interactive-data/2020-us-medical-school-faculty 


\section{Figures}

Figure 1: Integration of PERMA theory of well-being with intersectionalities mapped to GME trainee survey sections

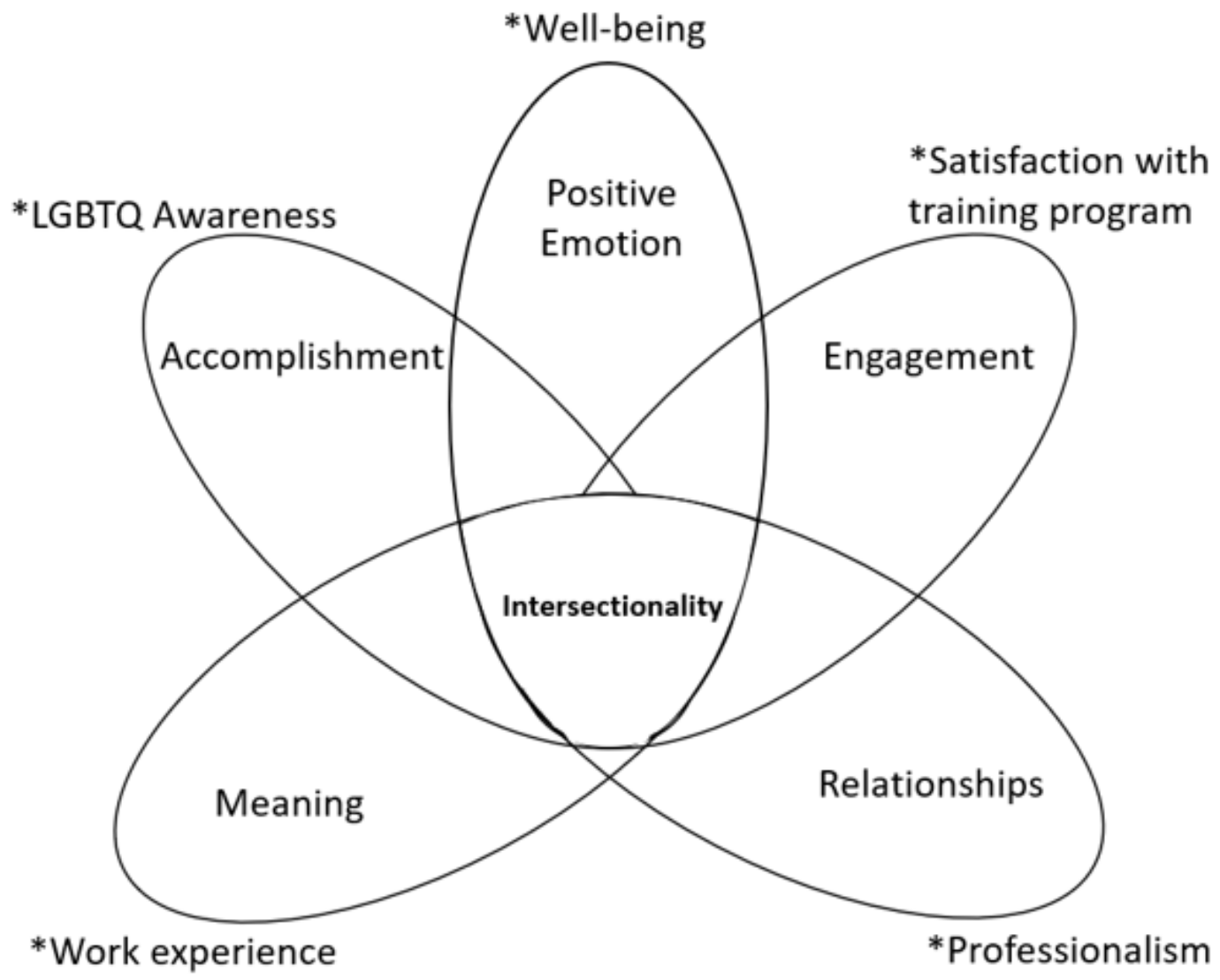

* Corresponding survey sections

Figure 1

See image above for figure legend

\section{Supplementary Files}

This is a list of supplementary files associated with this preprint. Click to download.

- ResidentGMEsurvey.pdf 